\title{
A NEURAL DECOMPOSITION OF VISUAL SEARCH USING VOXEL- BASED MORPHOMETRY
}

Glyn W. Humphreys and Magdalena Chechlacz

Department of Experimental Psychology

University of Oxford

South Parks Road

Oxford OX1 3UD, UK

Corresponding author: Magdalena Chechlacz, Department of Experimental Psychology, Oxford University, 9 South Parks Road, Oxford OX1 3UD

e-mail: magdalena.chechlacz@psy.ox.ac.uk

keywords: visual search, neuropsychological impairments, spatial working memory, neglect, voxel-based morphometry. 


\section{ABSTRACT}

The ability to search efficiently for visual targets amongst distractors can breakdown after a variety of brain lesions but the specific processes affected by the lesions are unclear. We examined search over space (conjunction search) and over time plus space (preview search) in a consecutive series of patients with acquired brain lesions. We also assessed performance on standard neuropsychological measures of visuo-spatial short-term memory (Corsi Block), sustained attention and memory updating (the contrast between forward and backward digit span) and visual neglect. Voxel-based morphometry (VBM) analyses revealed regions in the occipital (middle occipital gyrus), posterior parietal (angular gyrus) and temporal cortices (superior and middle temporal gyri extending to the insula), along with underlying white matter pathways, associated with poor search. Going beyond standard VBM analyses we then report correlation measures of structural damage in these regions and the independent neuropsychological measures of other cognitive functions. We find distinct patterns of correlation in areas linked to poor search suggesting that the areas play functionally different roles in search. We conclude that neuropsychological disorders of search can be linked to necessary and distinct cognitive functions, according to the site of lesion. 


\section{INTRODUCTION}

The ability to search efficiently over space and across time is critical for human survival. Studies of spatial search have very often focused on the detection of conjunctive targets, which cannot be distinguished from distractors on the basis of any single feature (e.g., Treisman, 1998; Treisman \& Gelade, 1980; Wolfe, 1994). Successful conjunction search depends on many factors the accurate coding of the features of the stimuli, grouping processes that help to separate targets and distractors (e.g, Duncan \& Humnphreys, 1989), the binding of the features making up each stimulus, the guidance of attention to items with features matching the 'template' for the target, the ability to sustain attention across time as search takes place, the accurate memory for items and locations that have already been searched (Klein, 1988), and so forth.

Brain imaging studies demonstrate that conjunction search recruits a fronto-parietal network associated with the control of visual attention (Corbetta, 1998; Corbetta \& Shulman, 2002; Coull, Walsh, Frith, \& Nobre, 2003; Mantini, Corbetta, Perrucci, Romani, \& Del Gratta, 2009; Nobre, Coull, Walsh, \& Frith, 2003; Shulman et al., 2003), over and above the sensory areas activated also when targets and distractors do differ in their component features. Donner et al. (2002) further reported the greater involvement of both parietal and frontal regions in conjunction search (e.g., the posterior intra-parietal sulcus and the frontal eye fields) along with some additional increases in activation within the occipital cortex. These imaging results are supported by neuropsychological data. For example, patients with posterior parietal cortex lesions have been shown to be selectively impaired at spatial search for conjunction targets, especially on the side of space contralateral to their lesion (Eglin, Robertson, \& Knight, 1989; Humphreys, Hodsoll, \& Riddoch, 2009; Riddoch \& Humphreys, 1987). This disruption, clinically associated with the neglect syndrome, may reflect several factors including poor orienting of attention to the affected side (Riddoch \& Humphreys, 1983), poor disengagement of attention from the ipsilesional side (Posner, Walker, Friedrich, \& Rafal, 1984), impaired perceptual binding (Friedman-Hill, 
Robertson, \& Treisman, 1995; Humphreys et al., 2009), impaired spatial working memory

(Chechlacz et al., 2014; Malhotra et al., 2005), and poor ability to sustain attention across time (Robertson, 2003). Exactly which of these factors is critical, and how the different factors link to the underlying brain lesions, is not fully specified however - partly because studies have tended to focus on relatively small patient groups selected by lesion site and partly because few studies report additional converging evidence to pin-point the functional nature of the deficit over and above the impairment in conjunction search. Nevertheless, since neuropsychological data can provide important information about which brain areas and functional processes are necessary to support search, more detailed analysis of why posterior parietal damage disrupts conjunction search should be theoretically informative. For example, feature integration theory (Treisman, 1998) proposes that the posterior parietal cortex is required for coding the locations of stimuli and that this is also critical for feature binding. Hence we would suggest that poor conjunction search after damage to this region would link also to impairments in spatial memory. The present study attempts to address this by analysing a group of patients with a heterogeneous set of lesions but then assessing how conjunction search correlates with damage to specific brain areas. In addition, converging evidence is presented on the performance of the patients on other tasks which depend on specific underlying processes, to help assess the functional role of the regions linked to poor search performance.

Preview search (Watson \& Humphreys, 1997) is a variant of conjunction search in which the sets of distractors are segmented across two time intervals, with the target appearing only with the second set (Watson \& Humphreys, 1997). This enables participants to use the time interval to segment the first distractors from the other items, so that search operates over time as well as space. Under preview conditions participants are normally able to ignore the early distractors, a process linked to activation of posterior parietal cortex (Allen, Humphreys, \& Matthews, 2008; Dent, Allen, Braithwaite, \& Humphreys, 2012) and associated behaviourally with 
distractor suppression (Allen et al., 2008; Watson \& Humphreys, 2000). However, in addition to distractor suppression efficient preview search may also be linked to sensitivity to new onsets (Donk \& Theeuwes, 2001; though see Humphreys, Olivers, \& Yoon, 2006) and to spatial working memory for the properties of the initial set of distractors (Allen et al., 2008). For example, preview search is disrupted by giving participants a secondary, working memory task during the preview task. Like conjunction search, preview search is also disrupted by damage to posterior parietal cortex (Humphreys et al., 2006; Olivers \& Humphreys, 2004), but the factors affected by posterior parietal damage have not been clearly identified. For example, is there an impaired ability to hold a spatial memory for the initial distractors, so that they are then difficult to suppress (Malhotra et al., 2005), is there poor temporal as well as spatial segmentation (Olivers \& Humphreys, 2004) or an impaired ability to suppress the early distractors (Fuentes \& Humphreys, 1996)? Indeed, if spatial memories are dependent upon the posterior parietal cortex and if they are crucial to both conjunction and preview search, then we would expect spatial memory to be impaired along with both search tasks after posterior parietal lesions. To date no studies have attempted to prise apart which of these processes may be responsible for the effects of parietal lesions on preview search.

In the present study we set out to examine the roles of different brain regions in spatial and spatio/temporal search based on conjunction versus preview visual search performance and using a novel extension of voxel-based morphometry (VBM; Ashburner \& Friston, 2000) analyses in brain lesioned patients. VBM can be used for lesion-symptom mapping in which neural changes are correlated with continuous measures of human performance across groups of patients (including those without as well as those with a behavioural deficit) to reveal the brain regions where lesions are associated with behavioural change (e.g., Rorden \& Karnath, 2004; Mechelli et al., 2005; Geva, Baron, Jones, Price, \& Warburton, 2012). VBM analyses have been used successfully to investigate a number of different neuropsychological deficits including spatial biases in visual neglect and extinction (e.g., Chechlacz et al., 2010; Chechlacz et al., 2013), visuo- 
spatial working memory impairments (Chechlacz et al., 2014), impairments in short-term memory (Leff et al., 2009), language problems (e.g., Geva et al., 2012; Leff et al., 2009; Rowan et al., 2007) and constructional apraxia (Chechlacz et al., 2014). Here we used VBM to assess the neural regions associated with impairments in spatial (conjunction) and spatio/temporal (preview) search, to test which brain regions are necessary for the accomplishment of these tasks.

Notably, VBM was extended by taking as regions of interest (ROIs) those brain areas associated with impaired search performance, but then examining the relations between structural changes in those areas and impairments in a set of independent cognitive tasks which assess specific processes which may contribute to visual search. In particular we measured visuospatial short-term memory, sustained attention and memory updating, and spatial representation and awareness across egocentric space and objects (see below for the tasks used for these independent measures). We then tested whether the ROIs linked to impaired search were associated with changes in these independent cognitive functions, to help identify the cognitive operations within the different areas. In contrast to other lesion-symptom mapping approaches such as voxel-based lesion-symptom mapping (VLSM), voxel-based morphometry (VBM) is based on continuous tissue integrity measures and therefore allows us to examine the association between performance on the independent measures of different cognitive processes and the underlying neural changes across the patient group. The VBM methods were also complemented by track-wise lesion deficits analyses enabling an assessment of damage to different voxels along the same white matter pathway across the entire brain (Thiebaut de Schotten et al., 2014).

\section{MATERIAL AND METHODS}

\section{Participants.}

Patients. Thirty-nine patients participated (34 males and 5 females), with ages ranging from 34 to 81 years (mean age 63.1 years). All patients had chronic acquired brain injury with clearly visible damage (> 9 months post diagnosis) and had no contraindications to MRI scanning. 
No other exclusion criteria were used. See Table 1 for full clinical and demographic data. All the patients were recruited from the panel of neuropsychological volunteers established in the Behavioural Brain Sciences Centre at the School of Psychology, University of Birmingham. All patients provided written informed consent in agreement with ethics protocols at the School of Psychology and Birmingham University Imaging Centre (BUIC).

Healthy Controls. For the lesion identification protocol (see below) we acquired T1weighted images from 100 healthy controls ( 55 males and 45 females, mean age 54.5 years, range 20-87) with no history of stroke, brain damage or neurological disorders. All the controls provided written informed consent in agreement with ethics protocols at the School of Psychology and BUIC. In addition to this, 20 control participants (10 male, 10 female), matched to age for the patients (age range 55-72, mean 65.3 years) took part in the visual search experiment.

\section{Cognitive assessment}

Visual Search. Participants were asked to carry out two computer-controlled visual search tasks. For both tasks the target was a blue $\mathrm{H}$ and the distractors were blue As and green $\mathrm{Hs}$. In the spatial search task (standard conjunction search) all the items were presented together and remained on the screen until the patient responded. In the spatio/temporal search task (preview search), the green $\mathrm{H}$ distractors were presented first for $1 \mathrm{sec}$ and followed after $1 \mathrm{sec}$ by the blue distractors and the target (see Figure 1A). After the appearance of the blue items the display remained on the screen until patients responded. The target (blue $\mathrm{H}$ ) was always present. The patients pressed the left mouse key as soon as they detected the target and then clicked the right or left mouse key to indicate whether the target had appeared on the left or right side of the display $^{1}$.

\footnotetext{
${ }^{1}$ With the current response set-up it is possible for participant to decide that a target must be on the opposite side if they cannot find it on one side of space. On the other hand, the procedure has been used successfully in several prior experiments (e.g., Olivers \& Humphreys, 2004), where the 
The stimuli were presented on a $38.1 \mathrm{~cm}$ monitor driven by a Dell PC using a resolution of $800 \times 600$ pixels. The displays were controlled using a purpose-written Turbo Pascal (Version 7.0) program, which also recorded RTs and responses. The viewing distance was approximately $75 \mathrm{~cm}$. The search displays were constructed by randomly plotting all search items in an 8x8 grid subtending approximately $8.3 \times 8.3$ degree in visual angle. The letters $\mathrm{A}$ and $\mathrm{H}$ were rectangular and 0.6 high $\times 0.4$ deg wide. The green (Commission Internationale d'Eclairage [CIE]: 0.23, 0.38) and blue colors (CIE: $0.26,0.32$ ) were chosen to match those used by Watson and Humphreys (1997) in the original study of preview search and they were roughly isoluminant (flicker test on the experimenter).

Each trial began with the appearance of a central fixation cross which remained on through the trial. Participants were asked to remain fixated throughout. After a 750 interval either the search display appeared (conjunction search) or the initial preview (preview search, green letters). In the preview condition this first display remained for $1 \mathrm{sec}$ and then the search items appeared (blue letters). In each case the search display remained on for $20 \mathrm{sec}$ or until the participant responded. The full display sizes were 4,8 and 16 (in the preview condition half of the items appeared in the first display). The target was always present and appeared randomly on the left or right of fixation.

All participants were able to use the mouse to respond. Feedback was provided on every trial (correct or incorrect). There were 120 trials per condition (40 per display size per participant, 20 target on the left and 20 target on the right). The search conditions were typically conducted in separate sessions with the order of the sessions counter-balanced over participants. To measure performance we derived an index of search efficiency based on (i) combining reaction times (RTs) with error in a processing efficiency measure (RT/proportion correct; Townsend \& Ashby, 1982) holds true here. 
and (ii) calculating the slope of the processing efficiency measure across the varying display sizes.

In addition to the visual search tasks, the patients also took part in a separate test session in which a number of other measures were taken based on visuo-spatial working memory, sustained attention/memory updating and the presence of unilateral neglect.

Visuo-spatial working memory. Visuo-spatial working memory was assessed using the Corsi Block Tapping test (Corsi, 1972). Testing was performed using a standard Corsi Block display (i.e., a wooden board with nine block/cubes mounted on the board with numbers visible only to examiner, placed at able height centred on the midline of the patient; Corsi, 1972; Lezak, 1995). The following instructions were given "This is a set of blocks. I am going to tap some of the blocks in a certain order with a pencil. Remember the order of the blocks as they are tapped, then as soon as I finish use your finger to tap the blocks in the same order". Subsequently, the examiner tapped the blocks (approximately one block per second) starting with only one block, followed by a sequence of two blocks, then three blocks and so on. The length of the tapped sequence was increased to test the capacity of the visuospatial short-term memory. Only the forward variant was used with the maximum number of 9 blocks per sequence and for each sequence length 3 trials were given. The testing was stopped if participant failed to reproduce any of the three sequences of a particular length. For each patient the block span was calculated indicating the length of the longest sequence correctly repeated by each patient.

Sustained attention/Memory updating. The ability to sustain attention and the ability to update items in memory were measured by contrasting digit span backwards with digit span forwards (see Robertson, 1990, for this argument). Patients were read out a series of digits in a random order, 1 every $3 \mathrm{sec}$, starting with 3 items and then increasing by 1 until the patient made 2 consecutive errors. The patients were asked to recall the items either forwards (in the order they received them) or backwards (in a reversed order). 20 patients started with the forwards digit span measure and 19 with the backwards span measure. 
Visual Neglect. Unilateral neglect symptoms were assessed using the Apples Cancellation task (Bickerton et al., 2011; Chechlacz et al., 2010; Humphreys et al., 2012). The Apples Cancellation task is similar to the gap detection task by Ota and colleagues and is designed to simultaneously measure egocentric and allocentric neglect (Ota, Fujii, Suzuki, Fukatsu, \& Yamadori, 2001). Participants were presented with a page (A4) in landscape orientation with 50 apples divided into 5 invisible columns, one middle, one near left, one far left, one near right and one far right. Each column contained 10 complete apples (targets) along with distractors i.e. apples with either a left or a right part missing (incomplete apples). Egocentric neglect was measured by whether patients miss targets (complete apples) on one side of the page. Allocentric neglect was measured by whether patients make false positive responses by cancelling distractors i.e. incomplete apples with gaps on a particular side.

\section{Neuroimaging assessment}

All patients and healthy controls were scanned at the Birmingham University Imaging Centre (BUIC) on a 3T Philips Achieva MRI system with 8-channel phased array SENSE head coil. We acquired anatomical scans using a sagittal T1-weighted sequence (sagittal orientation, TE/TR (echo time/repetition time)=3.8/8.4ms, voxel size $1 \times 1 \times 1 \mathrm{~mm}^{3}$ ). Taking into account that some patients presented with neurodegenerative changes and some with damage following carbon monoxide poisoning if possible we also acquired an additional scans using T2 FLAIR (Fluid Attenuated Inversion Recovery) sequence ( $T R=11000 \mathrm{~ms}, \mathrm{TE}=125 \mathrm{~ms}$, voxel size $0.45 \times 0.44 \times 2 \mathrm{~mm}^{3}$ ). While T1 scans were used in VBM analyses and lesion reconstruction, FLAIR scans were used to verify lesion demarcation (see below).

\section{Image pre-processing}

All T1 scans were converted and reoriented using MRICro (Chris Rorden, Georgia Tech, Atlanta, GA, USA). Pre-processing was done in SPM5 (Statistical Parametric Mapping, Welcome Department of Cognitive Neurology, London UK). The brain scans were transformed into the 
standard MNI (Montreal Neurological Institute) space using the unified-segmentation procedure (Ashburner \& Friston, 2005), which involves tissue classification based on the signal intensity in each voxel and on a-priori knowledge of the expected localization of grey matter (GM), white matter (WM), cerebrospinal fluid (CSF). We used here a modified segmentation procedure (see Seghier, Ramlackhansingh, Crinion, Leff, \& Price, 2008 for full details) to further improve tissue classification and spatial normalization of lesioned brains. This protocol was developed to resolve problems with misclassification of damaged tissue by including an additional prior for an atypical tissue class (an added "extra" class) to account for the "abnormal" voxels within lesions and thus allowing classification of the outlier voxels (Seghier et al., 2008). Following segmentation, we visually inspected each of the segmented scans to assess whether segmentation and normalisation was successful. Next, the segmented images were smoothed ${ }^{2}$ with $8 \mathrm{~mm}$ FWHM Gaussian filter to accommodate the assumption of random field theory used in the statistical analysis (Worsley, 2003). The choice of intermediate smoothing of $8 \mathrm{~mm}$ FWHM was previously shown to be optimal for lesion detection and further analysis of segmented images (Leff et al., 2009; Seghier et al., 2008; Stamatakis \& Tyler, 2005).

The pre-processed grey and white matter images were used for automated lesion identification using fuzzy clustering (Seghier et al., 2008) and in the voxel-based analyses to determine the relationships between lesion site and visual search performance. Previous work (e.g. Chechlacz et al., 2013; Leff et al., 2009; Price et al., 2010) has demonstrated that the modified segmentation protocol combined with VBM is successful in facilitating the understanding of brain behaviour relationships in neurological patients.

\section{Automated lesion identification}

\footnotetext{
${ }^{2}$ Prior to VBM analysis all segmented images are smoothed meaning that the intensity of the signal within each voxel is replaced by the weighted average of the surrounding voxels and the number of voxels averaged at each point is determined by the size of the smoothing kernel (here we used $8 \mathrm{~mm}$ FWHM Gaussian filter). 
Lesion maps from individual patients were reconstructed using a modified segmentation procedure (see above) and an outlier detection algorithm based on fuzzy clustering (see Seghier et al., 2008). This procedure identifies voxels that are different in the lesioned brain as compared to control healthy brains (here we used a set of scans from 100 healthy controls as described above) based normalised grey and white matter segments. The GM and WM outlier voxels are then combined into a single outlier image and thresholded to generate a binary map of the lesion (Seghier et al., 2008). The results of lesion reconstruction were verified against each patient's T1 and T2 FLAIR scans (if FLAIR scans were available). The binary lesion maps were used to calculate lesion volumes for each patient using Matlab R2012a (The MathWorks, Natick, MA, USA). The estimated lesion volumes of all individual patients were entered as covariate in the statistical analyses (see below). Finally, we overlaid the lesion maps from all 39 patients. The lesion overlay map was created to represent the spatial distribution of lesions in our group of patients.

\section{Voxel-based morphometry (VBM)}

To assess the relationship between WM and GM damage and visual search performance on a voxel-by voxel basis, we used VBM (Ashburner \& Friston, 2000) and carried out statistical analyses with SPM8 using smoothed GM and WM maps obtained from segmented scans from our patient sample (see above for the pre-processing protocol). We used parametric statistics within the framework of the general linear model (Kiebel \& Holmes, 2003) and the analyses for white (WM) and grey matter (GM) were carried out separately. We used three statistical models with different scores calculated based on visual search performance as the main covariate of interest: (i) overall conjunction search score, (ii) overall preview search score, and finally (iii) the field effects in conjunction and preview search (ipsi<contralesional) ${ }^{3}$. The GM and WM analyses were carried out separately. In each statistical model age, handedness, gender, type of diagnosis (stroke

\footnotetext{
${ }^{3}$ As all bilateral patients in the current study showed worse performance on one side in independent test of visuospatial attention (visual extinction test), we used that as a criterion for distinction between ipsi versus contralesional performance. 
or other) and time since diagnosis were included as covariates. We also entered as covariates the estimated lesion volume and controlled for the presence of visual field deficits and visuospatial deficits (spatial bias) indicated by left and right egocentric neglect and/or visual extinction (combined binary score for left and right visual field) as measured on the BCoS (The Birmingham Cognitive Screen) test battery (Humphreys et al., 2012). All these covariates ensured that we could control for various confounding factors that potentially might have affected cognitive performance on the search task.

We report only results that showed a significant effect at $p<0.05$ FWE cluster-level corrected for multiple comparisons with amplitude of voxels surviving of $p<0.001$ uncorrected across the whole brain and an extent threshold of 100 voxels. The brain coordinates are presented in standardized MNI space. The anatomical localization of the lesion sites within the GM was based on the Anatomical Automatic Labeling toolbox (AAL toolbox, Tzourio-Mazoyer et al., 2002), the Duvernoy Human Brain Atlas (Duvernoy, Cabanis, \& Vannson, 1991) and the Woolsey Brain Atlas (Woolsey, Hanaway, \& Gado, 2008). In order to localize the location of WM lesions in relation to specific white matter pathways we used the JHU (Johns Hopkins University) White matter tractography atlas (Hua et al., 2008) and the MRI Atlas of Human White Matter (Mori, 2005).

\section{Statistical analyses: Linking visual search deficits to cognitive components}

We next investigated the relationship between spatial and spatio/temporal search (respectively in the conjunction and preview search tasks) and the component processes assessed in the other neuropsychological tests (visuo-spatial working memory, sustained attention/memory updating, spatial biases in selection). Specifically, we examined the link between (i) grey and white matter density in the critical lesion areas for conjunction and preview search identified in the VBM analyses (see above) and (ii) behavioural performance on the Apples Cancellation task and the digit span and Corsi Block tests. The scores for the Apples cancellation task reflect the magnitude 
of egocentric neglect (numbers of target apples cancelled on the left vs. the right of the page) and allocentric neglect (the number of distractors cancelled according to whether they contained a gap on the left or right of each shape). Scores from the digit span task were used to calculate a ratio (composite measure/summed spans) for the relative drop in performance from forwards to backwards span, (forwards-backwards span/forwards + backwards span). Subsequent analysis showed that the Corsi Block span and composite measure from the digit span task were not correlated $(p=0.84)$, consistent with the measures tapping different aspects of cognition (visuospatial working memory i.e. Corsi Block and sustained attention/manipulating items in memory i.e. the drop in digit span measure). For both the neglect and digit span measures, a higher score indicates worse performance (more neglect or a relatively greater drop between forwards and backwards digit span conditions), while for the Corsi Block test a higher score (higher span) indicates better performance. To assess the relations to search, we first extracted tissue (grey and white matter) density taken from the principal eigenvariate of the voxels within an $8 \mathrm{~mm}$ radius of the peak voxel of each identified in VBM cluster and plotted against the above behavioural scores. The correlation between the plotted values was examined using Matlab R2012a (The MathWorks, Natick, MA, USA).

\section{Track-wise Lesion-Deficit analyses}

Voxel-based morphometry is based on mass-univariate approach and where the effects of behavioural predictors are assessed on each voxel independently (Ashburner \& Friston, 2000). While this approach is well suited for detecting lesion-behaviour relationships within discrete cortical subregions, the analysis has clear limitations when applied to white matter pathways, which often span across large distances within human brain, where spatially distant voxels may be part of the same anatomical tract. For this reason VBM analysis may not be sensitive enough to detect the relations between cognitive deficits and white matter changes; rather the evaluation of white matter change may require statistics derived at the tract level, which can take into account 
not separate voxels but strong relationship between distant voxels (Rudrauf, Mehta, \& Grabowski, 2008; Thiebaut de Schotten et al., 2008). Therefore, to supplement the VBM analysis and to assess the relations between white matter damage and visual search deficits we performed track-wise lesion deficit analyses based on an approach (Thiebaut de Schotten et al., 2014) utilizing diffusion tractography atlases of human white matter tracts (Thiebaut de Schotten, Dell'Acqua et al., 2011; Thiebaut de Schotten, Ffytche et al., 2011). By using the patients' reconstructed lesion maps (in MNI space), and the maps of white matter tracts from the above atlases (also in MNI space), we first evaluated the pattern of disconnection within all major white matter tracts (association, projection and commissural) for each individual patient. Our analyses were based on an atlas map of association pathways (inferior longitudinal fasciculus, inferior-fronto-occipital fasciculus, arcuate, cingulum, uncinate, superior longitudinal fasciculus segments I, II and III), commissural pathways (anterior commissure and corpus callosum) and projection pathways (fornix, internal capsule, optic radiations and cortico-spinal tract). All maps of white matter tracts represent a probability of a given voxel belonging to that tract and these maps were overlapped with the patients' lesion maps. We next calculated a continuous measure of the pathway disconnection by calculating the size of the overlap (in cubic centimetres) between each patient's lesion map and each thresholded (50\%) pathway map using Matlab 7.14/R2012a (The MathWorks, Natick, MA, USA). We used these continuous measures of white matter disconnections in the statistical trackwise lesion-deficit analyses based on linear regression. In the linear regression we entered lesion volume, age and each individual pathway disconnection measure as independent variables to test whether the disconnection within specific pathways (controlling for lesion volume and age) predicted visual search performance (a continuous behavioural measures). Matching the VBM analyses, we used three different scores for the linear regression assessments of visual search performance: (i) the overall conjunction search slope, (ii) overall preview search slope, and finally (iii) the field effects in conjunction and preview search (slope for ipsi<contralesional targets). The 
regression analyses were carried out separately for the left and right hemispheres. Each tract-wise lesion deficit analysis was subjected to Bonferroni correction for multiple comparisons ( $\alpha$ level; $p=0.004$ based on 14 tracts analysed). SPSS 21 software was used (IBM SPSS Statistics, NY, USA) to compute linear regressions in order to identify, which white matter pathways when damaged predicted the presence of visual search deficits.

It should be also noted that track-wise lesion deficit analysis had another advantage compared to VBM. In this analysis we used maps representing 3 discrete branches of the superior longitudinal fasciculus (SLF I-III) generated with spherical deconvolution tractography (Thiebaut de Schotten, Dell'Acqua et al., 2011; Thiebaut de Schotten, Ffytche et al., 2011). By contrast white matter atlases used in conjunction with VBM (Hua et al., 2008; Mori, 2005) are based solely on diffusion tensor imaging tractography, which cannot separate distinct anatomical branches within the SLF.

\section{RESULTS}

\section{Behaviour}

Figure 2 illustrates the behavioural performance and RT data used for the VBM and additional analyses based on the slopes of the search functions for the conjunction and preview conditions for all the patients included in the current study (plus also the results for the control participants).

The data for the patients and the age-matched controls were compared by calculating the slope of the search function for each participant and then entering into a mixed design ANOVA with search condition (preview vs. conjunction) and field (contra- vs. ipsilesional) as withinsubjects factors and group (patients vs. controls) as the between subjects factor. For half the control participants the left visual field was assigned to be contralesional and for the other half the right field was assigned to be contralesional. There were reliable main effects of search condition, 
field and group $(F(1,57)=66.57,18.42$ and 6.73 , all $p<0.015)$. There was a 2 -way interaction between field and group $(F(1,57)=14.04, p<0.001)$. There were higher slopes for conjunction compared with preview search and for patients compared with controls. In contrast with the controls, there were higher slopes in the contra-compared with the ipsilesional field for the patients.

The data for RTs were mirrored by the accuracy data (see Table 2).

\section{Grey matter substrates of spatial and temporal search}

The overall lesion distribution within both hemispheres for all patients is presented in Figure $1 \mathrm{~B}^{4}$.

We used VBM to investigate the neural substrates of visual search in our consecutive sample of neuropsychological patients. We found that deficits in overall performance on conjunction search were associated with grey matter damage within the right inferior parietal lobule centred on the angular gyrus (Figure 3A, Table 3), while deficits in overall performance on preview search were associated with bilateral damage within the middle occipital gyrus (MOG) as well as the right angular gyrus (Figure 4A, C, Table 3).

We next examined the field effect in conjunction and preview search. For conjunction search worse performance in the contralesional field (ipsi<contralesional) was associated with damage within right inferior parietal lobule centred on the supramarginal gyrus and extending into the postcentral gyrus as well as with damage within the middle temporal gyrus (MTG) and the superior temporal gyrus (STG) extending into the insula (Figure 5A, Table 3). We did not observe any reliable results for the field effect in preview search.

\footnotetext{
${ }^{4}$ Please note that the interpretation of lesion overlap map may be sometimes misleading as all voxels within the map are treated autonomously. For example, it is often the case that while several patients will have damage within the same brain area but as the area is comprised of many voxels, it would not be necessarily within exactly same voxels. Thus in the lesion overlap map, two or more voxels in immediate proximity within the same brain area could be labelled as damaged in only one or two patients but this could in fact refer to several different patients.
} 
Links to cognitive components. Grey matter density within both the left and right MOG clusters identified in the VBM analysis for preview search was strongly correlated with performance on the Corsi Block measure of visuo-spatial working memory (Figure 4B; right MOG, $r=0.37, p=0.02$; left MOG $r=0.4, p=0.01)^{5}$. Furthermore, grey matter density within the right angular gyrus region, found for both conjunction and preview search, was correlated with allocentric neglect $(r=-0.35, p=0.027 \text {; Figure } 4 D)^{5}$.

Taking the ROIs identified with the field effect in conjunction search we found that grey matter density changes within both the right inferior parietal lobule and the right temporal (MTG/STG) clusters were strongly correlated with the relative drop in performance between forwards and backwards digit span (Figure 5B). Furthermore, grey matter density within the right inferior parietal lobule cluster identified in the VBM analysis was strongly correlated with both egocentric and allocentric neglect measures from the Apples Cancellation test $(r=-0.33, p=0.04$ and $r=-0.47, p=0.003$ respectively; Figure $5 B)^{5}$.

\section{White matter substrates of spatial and spatio/temporal search}

VBM analyses and links to cognitive components. Overall performance on the conjunction search task was associated with white matter damage within the right inferior fronto-occipital fasciculus (IFOF) and internal capsule (Figure 3B, Table 4). Deficits in overall performance on preview search were associated with bilateral white matter damage within a region covering the IFOF and internal capsule but also the superior longitudinal fasciculus (SLF; Figure 4E, Table 4).

\footnotetext{
${ }^{5}$ Please note that, for the indices of neglect (allocentric and egocentric) and digit span (composite measure), a higher score indicates worse performance (more neglect or a relatively greater drop between forwards and backwards digit span conditions); in contrast for the Corsi Block test a higher score (higher span) indicates better performance. Thus we expected opposite corelations (positive versus negative) for the different indices. In each case we expected that higher grey or white matter density would be associated with better performance. However while for Corsi Block that would correspond to a positive correlation, for the neglect and digit span measures negative correlations should emerge. 
White matter density within the IFOF/internal capsule cluster was correlated with egocentric neglect $(r=-0.57, p=0.0001$; Figure $3 C)$ and Corsi Block performance $(r=0.47, p=0.002$; Figure $3 C)^{5}$. Interestingly Corsi Block performance was linked to white matter density within both the left and right white matter clusters (Figure $4 F$; right $r=0.37, p=0.02$; left $r=0.67, p=0.00001$ ) while right hemisphere damage within the cluster including the SLF, IFOF and internal capsule was associated with egocentric neglect $(r=-0.6 ; p=0.0001 \text {; Figure } 4 F)^{5}$.

We also examined the field effect in conjunction and preview search. For conjunction search worse performance in the contralesional field (ipsi<contralesional) was associated with white matter damage within a right hemisphere region including the SLF and internal capsule (Figure 5C, Table 4). We did not observe any reliable results for the field effect in preview search. These white matter lesions associated with the field effect in conjunction search did not correlate with performance on the other neuropsychological assessments.

Hodological track-wise lesion-deficit analyses. Subsequent to the VBM analyses, we adopted a hodological approach to understanding the contribution of white matter disconnections (Catani \& Mesulam, 2008a, 2008b; Rudrauf et al., 2008; Thiebaut de Schotten et al., 2008) based on linear regression performed to identify specific white matter pathways crucial to visual search. The linear regression analysis indicated that damage within the right IFOF was a predictor of poor overall conjunction search $(\beta=0.499 ; p=0.001)$ while damage within right SLF I $(\beta=0.486 ; p=0.002)$ was a predictor of poor overall performance on preview search. The analysis also revealed involvement of the right SLF II $(\beta=0.457 ; p=0.04$, preview search; $\beta=0.560 ; p=0.007$, conjunction search) and the right IFOF ( $\beta=0.391 ; p=0.02$, preview search) but these two results did not survive Bonferroni correction.

We next examined the consequence of white matter disconnections on the field effect in conjunction and preview search. For conjunction search worse performance in the contralesional field (ipsi<contralesional) was linked to disconnection within right SLF II $(\beta=0.942 ; p=0.0001)$, right 19 
SLF III ( $\beta=0.986 ; p=0.0001)$ and right corticospinal tract $(\beta=0.717 ; p=0.001)$. Importantly, while VBM analysis failed to identify the link between white matter disconnection and field effect in preview search, the hodological analysis indicated that, for preview search, worse performance in the contralesional field resulted from damage within right SLF II $(\beta=0.785 ; p=0.0001)$ and right SLF III $(\beta=0.671 ; p=0.003)$.

We did not observe any reliable results for the link between disconnection within left hemisphere pathways and performance on either conjunction or preview search.

\section{DISCUSSION}

We used VBM analysis to assess lesion-symptom relations linked to impairments in spatial and temporal search (conjunction and preview search). Overall our patient group showed slower search than controls, relatively worse performance in conjunction compared with preview search, and stronger effects of visual field on search. Our lesion-symptom analysis, using voxel-based morphometry (VBM), showed that poor overall spatial and temporal search was associated with lesions to the right angular gyrus, with temporal search (in the preview condition) also linked to damage to the bilateral middle occipital gyrus (MOG). In addition, our tract-based analysis of white matter damage revealed that the overall deficits in conjunction search were associated with damage to the right inferior fronto-occipital fasciculus (IFOF), while damage to the superior longitudinal fasciculus, specifically segment I (SLF I) was linked to overall poor performance in preview search. Furthermore, spatial deficits (field effects) within conjunction search were found after grey matter damage within the right inferior parietal lobule (centred on the supramarginal gyrus and extending into the postcentral gyrus) as well as damage within the middle temporal gyrus (MTG) and the superior temporal gyrus (STG) extending into the insula. Spatial deficits in both conjunction and preview search were linked to damage in the right SLF (SLF II and SLF III). As indicated in Table 1, our group of patients included individuals with right, left and bilateral lesions. 
Taking into account the relatively small number of patients with unilateral left hemisphere lesions $(n=8)$ and the overall lesion distribution (Figure 1B), it is plausible that the results of the VBM analysis were somewhat driven by patients with right hemisphere damage.

Both spatial (conjunction) and spatio/temporal (preview) search involve several factors including visual orienting of attention, target selection, memory for and suppression of previously attended distractors, and sustaining of attention and updating of memory over time. This means that it is difficult to conclude exactly which factors are disrupted in relation to the brain lesions, taking search performance as a whole. To advance our analysis, we extended the VBM approach by assessing the performance of patients on other neuropsychological tests sensitive to visuospatial working memory (Corsi Block), orienting and target selection across different spatial representations (ego- and allocentric neglect), and sustaining and updating memory across time (the drop in backwards relative to forwards digit span). We then evaluated whether the changes in grey and white matter in the regions of interest correlated with performance on these independent tasks.

\section{Overall deficits in visual search}

Damage to the right angular gyrus was linked to overall impairments in both spatial and spatio/temporal search and these lesions strongly correlated with the presence of allocentric neglect in the patients, but not with other cognitive components (e.g., visuo-spatial memory, sustaining and updating of memory, egocentric neglect). Allocentric neglect putatively reflects a problem in spreading attention to both sides of a selected spatial region (e.g., both sides of a target object) and can be found irrespective of the position of the selected spatial region with the left and right visual fields (Bickerton et al., 2011; Caramazza \& Hillis, 1990; Chechlacz et al., 2010). Here we suggest that overall problems in spatial and temporal search, affecting target selection in both left and right fields, may stem from poor attention within selected spatial regions and this is 
associated with lesions of the right angular gyrus. Reduced attention within selected spatial regions will in turn reduce the ability to discriminate targets from distractors and generate larger effects of distractor display size on search. It is of interest that the effects of right hemisphere damage here are associated with non-spatial (overall) deficits in search and this is consistent with this region of the right hemisphere having an over-arching role in selection within both the left and right sides of space (Corbetta \& Shulman, 2002).

Overall deficits in preview search were not only related to lesions affecting the angular gyrus but also damage to the middle occipital gyrus (MOG). Neural changes within the MOG were also associated with reductions in visuo-spatial memory on the Corsi Block test. Previous results have shown that preview search is dependent on the ability to encode in memory a representation of the initial distractors, which can subsequently be suppressed (see Allen et al., 2008; Emrich, Al-Aidroos, Pratt, \& Ferber, 2010; Humphreys, Watson, \& Jolicoeur, 2002). The correlation between poor visuo-spatial working memory and impaired preview search here suggests that the MOG may be critical in either encoding representations of the initial distractors or in suppressing these items so the items no longer compete for selection. This proposal is supported by functional imaging studies showing the selective activation of the MOG in preview relative to conjunction search tasks (e.g., Allen et al., 2008; Dent et al., 2012). There is also evidence for the activation of the MOG in studies of visuospatial working memory (Nemmi, Boccia, Piccardi, Galati, \& Guariglia, 2013; Toepper et al., 2010). The emergence of this result for preview rather than conjunction search also indicates that the MOG may be particularly involved in the parallel coding of a visual memory representation (found in preview search) rather than on the memory representation being coded serially (which is more likely in conjunction search, typically associated with larger search slopes than preview search; see Watson \& Humphreys, 1997).

Overall performance deficits in both spatial and spatio/temporal search were also linked to white matter damage within pathways including the IFOF, the internal capsule and SLF 
(predominantly in preview search). While the VBM analysis of white matter pointed to bilateral lesions within these regions, the hodological tract-based analysis highlighted the right hemisphere and pointed to a finer-grained breakdown. Specifically poor conjunction search was linked to damage to the IFOF and impaired preview search to damage to SLF I. Both IFOF and SLF have been previously identified as part of the fronto-parietal network associated with spatial attention, visuospatial orienting, visual selection and spatial working memory (Aralasmak et al., 2006; Schmahmann \& Pandya, 2006; Schmahmann et al., 2007). Interestingly, we found here that bilateral damage within the IFOF and SLF regions identified in VBM, associated with poor visual search, was also linked to reduced Corsi Block span, perhaps because good encoding of visual stimuli into memory is contingent upon efficient transcription of information across cortical brain regions subserved by the affected the fibre tracts. This could then impact on both conjunction and preview search if there are effects on serial as well as parallel encoding to memory. Importantly this is consistent with the notion that these pathways may support the top-down control of spatial representations in order to both detect stimuli in the environment and to actively maintain their representations in memory (Chechlacz et al., 2014; Makris et al., 2005; Petrides \& Pandya, 2006; Schmahmann \& Pandya, 2006; Schmahmann, Smith, Eichler, \& Filley, 2008). There were also associations between damage to these white matter regions in the right hemisphere and egocentric neglect, with patients showing more neglect when there were greater reductions in white matter tissue. These data fit with the argument that neglect can result from disconnections within the right hemisphere (Bartolomeo, Thiebaut de Schotten, \& Doricchi, 2007; Doricchi, Thiebaut de Schotten, Tomaiuolo, \& Bartolomeo, 2008; Thiebaut de Schotten et al., 2014), biasing attentional orienting to the left side. Note that the VBM on overall deficits in conjunction and preview search here took into account the degree of neglect on clinical tests, so the present result does not reflect the presence of neglect errors themselves during search. However it may still be that patients can be abnormally slow in searching and detecting targets on the contralesional side, 
even when this is not manifest in neglect errors on search under the relatively prolonged viewing conditions in the experiment. This bias in selection could nevertheless generate neglect under more challenging clinical conditions. The link to egocentric neglect here suggests a problem particular in attentional scanning over and above other deficits.

It is worth noting too that the hodological track-wise lesion analysis pointed to the link between SLF I damage and preview search. This tract links regions within the dorsal attention network and has been linked to controlling the ability to spatially orient attention to visual targets (Corbetta \& Shulman, 2002; Thiebaut de Schotten, Dell'Acqua et al., 2011). The present evidence implicating SLF I more strongly in preview than conjunction search suggests that SLF I may support processes particularly involved in preview search - for example the parallel coding of items in memory and the application of top-down inhibition across distractors. In contrast, the link between IFOF damage and conjunction search highlights the role of this tract in serial visual search - for example, in the spatial direction of attention and/or the serial encoding of items into memory.

\section{Field deficits in visual search}

We also examined field deficits in search. Here lesions of the inferior right parietal cortex and the middle and superior temporal gyri were correlated with relatively poor performance on conjunction search for contralesional targets. Within these regions of interest there were correlations with allo- and egocentric neglect (inferior parietal cortex) and sustained attention/memory updating (MTG/STG). The link of both allo- and egocentric neglect to damage to the inferior parietal cortex fits with classic accounts that associate the inferior right parietal lobe with neglect. Though there is evidence for dissociations between allo- and egocentric neglect from both behavioural (Bickerton et al., 2011) and neuroimaging data (Chechlacz et al., 2010; Verdon, Schwartz, Lovblad, Hauert, \& Vuilleumier, 2010), Chechlacz et al. (2010) have also argued that the inferior parietal cortex/temporo-parietal junction serves as a hub for orienting attention 
to both egocentric and allocentric spatial representations. If that is the case then damage to this region will be associated with both forms of neglect as well as spatial biases in search, as we observed. The impairment in sustained attention/attentional updating, however, was also linked to lesions of the MTG/STG (extending to the insula). Though it has proved a controversial point in the literature (e.g., contrast Karnath, 2001; Karnath, Fruhmann Berger, Kuker, \& Rorden, 2004; Karnath, Fruhmann Berger, Zopf, \& Kuker, 2004 with Mort et al., 2004; Mort et al., 2003), there is now considerable evidence that left unilateral neglect can be found after lesions of the MTG/STG (e.g., see Chechlacz et al., 2010; Chechlacz, Rotshtein, Roberts et al., 2012; Karnath, Fruhmann Berger, Kuker et al., 2004; Karnath, Rennig, Johannsen, \& Rorden, 2011; for a further review see also Chechlacz, Rotshtein, \& Humphreys, 2012; Karnath \& Rorden, 2012). This argument is also supported by the association we report between MTG/STG damage and the field effects in conjunction search. Interestingly lesions of the MTG/STG cluster also correlated with clinical measures of sustained attention/memory updating (the drop in performance on backwards relative to forwards digit span; Robertson, 1990). This for the first time points to the role of these more anterior regions in the neglect syndrome, namely in the sustaining of attention and updating of memory (e.g., as consecutive items are loaded into working memory as a set of numbers is transformed from back to front). It is perhaps not surprising then that the more anterior regions are also associated with egocentric (rather than allocentric) neglect, using tests where patients must scan attention across a spatial region in order plan and transverse a search path, since the scanning operation will challenge the ability to sustain attention and update previously searched locations.

The evidence for field deficits in search was clearer for spatial than for temporal search in our VBM analyses (there were no cortical regions significantly associated with field effects in preview search). This is perhaps not surprising given that spatial search is the more difficult task for normal participants (Watson \& Humphreys, 1997) and will place greater demands on sustained 
attention and memory updating processes. It is nevertheless worthwhile noting that an apparent deficit linked to poor sustained attention did not modulate factors included in preview search such as the ability to orientation to the onset of the new search display and to segment the old and new displays. This suggests that the ability to sustain attention over time (measured in backwards span) can dissociate from temporal orienting and segmentation over shorter time periods (e.g., in preview search). It should be noted however that while the VBM approach indicated only a significant association between contralesional field deficits in conjunction (spatial) search and white matter damage within a region including the right SLF, the more sophisticated hodological analysis revealed that disconnections within two segments of SLF within right hemisphere, SLF II and SLF III were critical predictors of contralesional field deficits in both spatial (conjunction) and temporal (preview) search. Disconnections of the SLF within the right hemisphere have been implicated in visual neglect (e.g., Bartolomeo et al., 2007; Chechlacz et al., 2010; Doricchi \& Tomaiuolo, 2003) and in particular the damage within the right SLF II (Thiebaut de Schotten, Dell'Acqua et al., 2011; Thiebaut de Schotten et al., 2014). This has been argued to reflect a functional role of the SLF II in linking the dorsal and ventral attention networks involved in spatial search and target recognition (Corbetta \& Shulman, 2002, 2011; Thiebaut de Schotten, Dell'Acqua et al., 2011; Thiebaut de Schotten et al., 2014). The SLF III in contrast has been identified as pathway linking regions within the ventral attentional network underlying visual target driven capture of spatial attention (Corbetta, Kincade, Ollinger, McAvoy, \& Shulman, 2000; Corbetta \& Shulman, 2002). Our results are consistent with both biased search and biased attentional selection of targets leading to field effects in search over space and time.

\section{Acknowledgements}

This work was supported by funding from the NIHR Oxford cognitive health Clinical Research Facility and the Stroke Association (GWH) and from the British Academy (MC). 


\section{FIGURE LEGENDS}

Figure 1. (A) Visual Search Task: example of displays (size 16) used for conjunction search (spatial search task; left panel) and preview search (temporal search task; right panel); see Material and Methods section for full description. (B) Lesion overlap map representing the spatial distribution of lesions among all 39 patients included in the current study. Lesion maps from individual patients were reconstructed based on Seghier et al. (2008) method; see Materials and Methods section for details. The lesion overlap map is shown on seven axial slices in standard MNI space with given MNI Z-coordinates of the presented axial sections. The colour bar shows the number of patients with a lesion within particular voxel (range 1-39).

Figure 2. Mean RTs in preview and conjunction search for control participants and patients. Data for the patients are separated according to whether targets fell on the contra- or ipsilesional side of space. For patients with a bilateral lesion, the contralesional side was selected on the basis of which side showed the worst performance.

Figure 3. Conjunction Search: VBM analyses. (A) Grey and (B) white matter substrates of overall deficits on conjunction search. The lesioned areas associated with deficits are coloured according to the level of significance in the VBM analyses, where brighter colours represent higher t-values. (C) Correlation between white matter damage (changes in tissue density) associated with poor performance on conjunction search and performance on other neuropsychological tests. White matter density values were taken from the principal eigenvariate (i.e. a summary of values) of the cluster identified in VBM analysis. AG, angular gyrus; IFOF, inferior fronto-occipital fasciculus; INTC, internal capsule.

Figure 4. Preview Search: VBM analyses. (A, C) Grey and (E) white matter substrates of overall deficits on preview search. The lesioned areas associated with deficits are coloured according to the level of significance in the VBM analyses, where brighter colours represent higher t-values. 
Correlation between (B, D) grey and (F) white matter damage (changes in tissue density) associated with poor performance on preview search and performance on other neuropsychological tests. Grey and white matter density values were taken from the principal eigenvariate (i.e. a summary of values) of the cluster identified in VBM analysis. AG, angular gyrus; IFOF, inferior fronto-occipital fasciculus; INTC, internal capsule; MOG, middle occipital gyrus; SLF, superior longitudinal fasciculus.

Figure 5. Field effect in conjunction search (ipsi<contralesional): VBM analyses. (A) Grey and (C) white matter substrates of poor performance in the contralesional field (ipsi<contralesional) in the conjunction search. The lesioned areas associated with deficits are coloured according to the level of significance in the VBM analyses, where brighter colours represent higher t-values. Correlation between (B) grey matter damage (changes in tissue density) associated with poor performance in the contralesional field in the conjunction search and performance on other neuropsychological tests. Grey matter density values were taken from the principal eigenvariate (i.e. a summary of values) of the cluster identified in VBM analysis. INTC, internal capsule; MTG, middle temporal gyrus extending into insula; SLF, superior longitudinal fasciculus; SMG, supramarginal gyrus extending into postcentral gyrus; STG, superior temporal gyrus. 


\section{REFERENCES}

Allen, H. A., Humphreys, G. W., \& Matthews, P. M. (2008). A neural marker of content-specific active ignoring. J Exp Psychol Hum Percept Perform, 34, 286-297.

Aralasmak, A., Ulmer, J. L., Kocak, M., Salvan, C. V., Hillis, A. E., \& Yousem, D. M. (2006). Association, commissural, and projection pathways and their functional deficit reported in literature. $J$ Comput Assist Tomogr, 30, 695-715.

Ashburner, J., \& Friston, K. J. (2000). Voxel-based morphometry--the methods. Neuroimage, 11, 805-821.

Ashburner, J., \& Friston, K. J. (2005). Unified segmentation. Neuroimage, 26, 839-851.

Bartolomeo, P., Thiebaut de Schotten, M., \& Doricchi, F. (2007). Left unilateral neglect as a disconnection syndrome. Cerebral Cortex, 17, 2479-2490.

Bickerton, W. L., Samson, D., Williamson, J., \& Humphreys, G. W. (2011). Separating Forms of Neglect Using the Apples Test: Validation and Functional Prediction in Chronic and Acute Stroke. Neuropsychology, 25, 567-580.

Caramazza, A., \& Hillis, A. E. (1990). Spatial representation of words in the brain implied by studies of a unilateral neglect patient. Nature, 346, 267-269.

Catani, M., \& Mesulam, M. (2008a). The arcuate fasciculus and the disconnection theme in language and aphasia: history and current state. Cortex, 44, 953-961.

Catani, M., \& Mesulam, M. (2008b). What is a disconnection syndrome? Cortex, 44, 911-913.

Chechlacz, M., Novick, A., Rotshtein, P., Bickerton, W. L., Humphreys, G. W., \& Demeyere, N. (2014). The Neural Substrates of Drawing: A Voxel-based Morphometry Analysis of Constructional, Hierarchical, and Spatial Representation Deficits. J Cogn Neurosci, 1-15.

Chechlacz, M., Rotshtein, P., Bickerton, W. L., Hansen, P. C., Deb, S., \& Humphreys, G. W. (2010). Separating neural correlates of allocentric and egocentric neglect: Distinct cortical sites and common white matter disconnections. Cognitive Neuropsychology, 27, 277-303.

Chechlacz, M., Rotshtein, P., Hansen, P. C., Deb, S., Riddoch, M. J., \& Humphreys, G. W. (2013). The central role of the temporo-parietal junction and the superior longitudinal fasciculus in supporting multiitem competition: evidence from lesion-symptom mapping of extinction. Cortex, 49, 487-506.

Chechlacz, M., Rotshtein, P., \& Humphreys, G. W. (2012). Neuroanatomical Dissections of Unilateral Visual Neglect Symptoms: ALE Meta-Analysis of Lesion-Symptom Mapping. Front Hum Neurosci, 6, 230.

Chechlacz, M., Rotshtein, P., Roberts, K. L., Bickerton, W. L., Lau, J. K., \& Humphreys, G. W. (2012). The prognosis of allocentric and egocentric neglect: evidence from clinical scans. PLoS One, 7, e47821.

Chechlacz, M., Rotshtein, P., Humphreys, G.W. (2014). Neuronal substrates of Corsi Block span: lesionsymptom mapping analyses in relation to attentional competition and spatial bias. Neuropsychologia, 64: 240-251.

Corbetta, M. (1998). Frontoparietal cortical networks for directing attention and the eye to visual locations: identical, independent, or overlapping neural systems? Proc Natl Acad Sci U S A, 95, 831-838.

Corbetta, M., Kincade, J. M., Ollinger, J. M., McAvoy, M. P., \& Shulman, G. L. (2000). Voluntary orienting is dissociated from target detection in human posterior parietal cortex. Nat Neurosci, 3, 292-297.

Corbetta, M., \& Shulman, G. L. (2002). Control of goal-directed and stimulus-driven attention in the brain. Nat Rev Neurosci, 3, 201-215.

Corbetta, M., \& Shulman, G. L. (2011). Spatial neglect and attention networks. Annu Rev Neurosci, 34, 569599.

Corsi, P.M. (1972). Human memory and the medial temporal region of the brain. Dissertation Abstracts International, 34, 819B

Coull, J. T., Walsh, V., Frith, C. D., \& Nobre, A. C. (2003). Distinct neural substrates for visual search amongst spatial versus temporal distractors. Brain Res Cogn Brain Res, 17, 368-379.

Dent, K., Allen, H. A., Braithwaite, J. J., \& Humphreys, G. W. (2012). Parallel distractor rejection as a binding mechanism in search. Front Psychol, 3, 278.

Donk, M., \& Theeuwes, J. (2001). Visual marking beside the mark: prioritizing selection by abrupt onsets. Percept Psychophys, 63, 891-900. 
Donner, T. H., Kettermann, A., Diesch, E., Ostendorf, F., Villringer, A., \& Brandt, S. A. (2002). Visual feature and conjunction searches of equal difficulty engage only partially overlapping frontoparietal networks. Neuroimage, 15, 16-25.

Doricchi, F., Thiebaut de Schotten, M., Tomaiuolo, F., \& Bartolomeo, P. (2008). White matter (dis)connections and gray matter (dys)functions in visual neglect: gaining insights into the brain networks of spatial awareness. Cortex, 44, 983-995.

Doricchi, F., \& Tomaiuolo, F. (2003). The anatomy of neglect without hemianopia: a key role for parietalfrontal disconnection? Neuroreport, 14, 2239-2243.

Duncan, J., \& Humphreys, G. W. (1989). Visual search and stimulus similarity. Psychol Rev, 96, 433-458.

Duvernoy, H. M., Cabanis, E. A., \& Vannson, J. L. (1991). The Human brain : surface, three-dimensional sectional anatomy and MRI. Wien: Springer-Verlag.

Eglin, M., Robertson, L. C., \& Knight, R. T. (1989). Visual search performance in the neglect syndrome. J Cogn Neurosci, 1, 372-385.

Emrich, S. M., Al-Aidroos, N., Pratt, J., \& Ferber, S. (2010). Finding memory in search: the effect of visual working memory load on visual search. Q J Exp Psychol (Hove), 63, 1457-1466.

Friedman-Hill, S. R., Robertson, L. C., \& Treisman, A. (1995). Parietal contributions to visual feature binding: evidence from a patient with bilateral lesions. Science, 269, 853-855.

Fuentes, L. J., Humphreys, G.W. (1996). On the Processing of "Extinguished" Stimuli in Unilateral Visual Neglect: An Approach Using Negative Priming. Cognitive Neuropsychology, 13, 111-136.

Geva, S., Baron, J. C., Jones, P. S., Price, C. J., \& Warburton, E. A. (2012). A comparison of VLSM and VBM in a cohort of patients with post-stroke aphasia. Neuroimage Clin, 1, 37-47.

Hua, K., Zhang, J. Y., Wakana, S., Jiang, H. Y., Li, X., Reich, D. S., et al. (2008). Tract probability maps in stereotaxic spaces: Analyses of white matter anatomy and tract-specific quantification. Neuroimage, 39, 336-347.

Humphreys, G.W., Bickerton, W.L., Samson, D., Riddoch, M.J. (2012). The Birmingham Cognitive Screen (BCoS). (1st ed.). London: Psychology Press.

Humphreys, G. W., Hodsoll, J., \& Riddoch, M. J. (2009). Fractionating the binding process: neuropsychological evidence from reversed search efficiencies. J Exp Psychol Hum Percept Perform, 35, 627-647.

Humphreys, G. W., Olivers, C. N., \& Yoon, E. Y. (2006). An onset advantage without a preview benefit: neuropsychological evidence separating onset and preview effects in search. J Cogn Neurosci, 18, 110-120.

Humphreys, G. W., Watson, D. G., \& Jolicoeur, P. (2002). Fractionating the preview benefit in search: dualtask decomposition of visual marking by timing and modality. J Exp Psychol Hum Percept Perform, $28,640-660$.

Karnath, H. O. (2001). New insights into the functions of the superior temporal cortex. Nature Review Neuroscience, 2, 568-576.

Karnath, H. O., Fruhmann Berger, M., Kuker, W., \& Rorden, C. (2004). The anatomy of spatial neglect based on voxelwise statistical analysis: a study of 140 patients. Cerebral Cortex, 14, 1164-1172.

Karnath, H. O., Fruhmann Berger, M., Zopf, R., \& Kuker, W. (2004). Using SPM normalization for lesion analysis in spatial neglect. Brain, 127, E10; author reply E11.

Karnath, H. O., Rennig, J., Johannsen, L., \& Rorden, C. (2011). The anatomy underlying acute versus chronic spatial neglect: a longitudinal study. Brain, 134, 903-912.

Karnath, H. O., \& Rorden, C. (2012). The anatomy of spatial neglect. Neuropsychologia, 50, 1010-1017.

Kiebel, S. \& Holmes, A. The General Linear Model. (2003). In Frackowiak, R.S.J, Friston, K.J., Frith, C., Dolan, R., Price, C.J., Zeki, S., Ashburner, J., \& Penny, W.D. (Eds). Human Brain Function (2nd edition; pp. 725-760). London: Academic Press.

Klein, R. (1988). Inhibitory tagging system facilitates visual search. Nature, 334, 430-431.

Leff, A. P., Schofield, T. M., Crinion, J. T., Seghier, M. L., Grogan, A., Green, D. W., et al. (2009). The left superior temporal gyrus is a shared substrate for auditory short-term memory and speech comprehension: evidence from 210 patients with stroke. Brain, 132, 3401-3410.

Lezak, M. D. (1995). Neuropsychological assessment (3rd ed.). New York ; Oxford: Oxford University Press. 
Makris, N., Kennedy, D. N., Mclnerney, S., Sorensen, A. G., Wang, R., Caviness, V. S., Jr., et al. (2005). Segmentation of subcomponents within the superior longitudinal fascicle in humans: a quantitative, in vivo, DT-MRI study. Cereb Cortex, 15, 854-869.

Malhotra, P., Jager, H. R., Parton, A., Greenwood, R., Playford, E. D., Brown, M. M., et al. (2005). Spatial working memory capacity in unilateral neglect. Brain, 128, 424-435.

Mantini, D., Corbetta, M., Perrucci, M. G., Romani, G. L., \& Del Gratta, C. (2009). Large-scale brain networks account for sustained and transient activity during target detection. Neuroimage, 44, 265-274.

Mori, S. (2005). MRI atlas of human white matter. Amsterdam ; Oxford: Elsevier.

Mechelli, A., Price, C.J., Friston, K.J., Ashburner, J. (2005). Voxel-based morphometry of the human brain: methods and applications. Current Medical Imaging Reviews, 1, 105-113.

Mort, D. J., Malhotra, P., Mannan, S. K., Pambakian, A., Kennard, C., \& Husain, M. (2004). Reply to: Using SPM normalization for lesion analysis in spatial neglect. Brain, 127, E11-U14.

Mort, D. J., Malhotra, P., Mannan, S. K., Rorden, C., Pambakian, A., Kennard, C., et al. (2003). The anatomy of visual neglect. Brain, 126, 1986-1997.

Nemmi, F., Boccia, M., Piccardi, L., Galati, G., \& Guariglia, C. (2013). Segregation of neural circuits involved in spatial learning in reaching and navigational space. Neuropsychologia, 51, 1561-1570.

Nobre, A. C., Coull, J. T., Walsh, V., \& Frith, C. D. (2003). Brain activations during visual search: contributions of search efficiency versus feature binding. Neuroimage, 18, 91-103.

Olivers, C. N., \& Humphreys, G. W. (2004). Spatiotemporal segregation in visual search: evidence from parietal lesions. J Exp Psychol Hum Percept Perform, 30, 667-688.

Ota, H., Fujii, T., Suzuki, K., Fukatsu, R., \& Yamadori, A. (2001). Dissociation of body-centered and stimuluscentered representations in unilateral neglect. Neurology, 57, 2064-2069.

Petrides, M., \& Pandya, D. N. (2006). Efferent association pathways originating in the caudal prefrontal cortex in the macaque monkey. Journal of Comparative Neurology, 498, 227-251.

Posner, M. I., Walker, J. A., Friedrich, F. J., \& Rafal, R. D. (1984). Effects of parietal injury on covert orienting of attention. J Neurosci, 4, 1863-1874.

Price, C. J., Crinion, J. T., Leff, A. P., Richardson, F. M., Schofield, T. M., Prejawa, S., et al. (2010). Lesion sites that predict the ability to gesture how an object is used. Archives Italiennes De Biologie, 148, 243258.

Riddoch, M. J., \& Humphreys, G. W. (1983). The effect of cueing on unilateral neglect. Neuropsychologia, 21, 589-599.

Riddoch, M. J., \& Humphreys, G. W. (1987). A case of integrative visual agnosia. Brain, 110 ( Pt 6), 14311462.

Robertson, I. H. (1990). Digit span and visual neglect: a puzzling relationship. Neuropsychologia, 28, 217222.

Robertson, L. C. (2003). Binding, spatial attention and perceptual awareness. Nat Rev Neurosci, 4, 93-102.

Rorden, C., \& Karnath, H. O. (2004). Using human brain lesions to infer function: a relic from a past era in the fMRI age? Nat Rev Neurosci, 5, 813-819.

Rowan, A., Vargha-Khadem, F., Calamante, F., Tournier, J. D., Kirkham, F. J., Chong, W. K., et al. (2007). Cortical abnormalities and language function in young patients with basal ganglia stroke. Neuroimage, 36, 431-440.

Rudrauf, D., Mehta, S., \& Grabowski, T. J. (2008). Disconnection's renaissance takes shape: Formal incorporation in group-level lesion studies. Cortex, 44, 1084-1096.

Schmahmann, J. D., \& Pandya, D. N. (2006). Fiber pathways of the brain. Oxford ; New York: Oxford University Press.

Schmahmann, J. D., Pandya, D. N., Wang, R., Dai, G., D'Arceuil, H. E., de Crespigny, A. J., et al. (2007). Association fibre pathways of the brain: parallel observations from diffusion spectrum imaging and autoradiography. Brain, 130, 630-653.

Schmahmann, J. D., Smith, E. E., Eichler, F. S., \& Filley, C. M. (2008). Cerebral white matter: neuroanatomy, clinical neurology, and neurobehavioral correlates. Ann N Y Acad Sci, 1142, 266-309.

Seghier, M. L., Ramlackhansingh, A., Crinion, J., Leff, A. P., \& Price, C. J. (2008). Lesion identification using unified segmentation-normalisation models and fuzzy clustering. Neuroimage, 41, 1253-1266. 
Shulman, G. L., McAvoy, M. P., Cowan, M. C., Astafiev, S. V., Tansy, A. P., d'Avossa, G., et al. (2003). Quantitative analysis of attention and detection signals during visual search. J Neurophysiol, 90, 3384-3397.

Stamatakis, E. A., \& Tyler, L. K. (2005). Identifying lesions on structural brain images--validation of the method and application to neuropsychological patients. Brain and Language, 94, 167-177.

Thiebaut de Schotten, M., Dell'Acqua, F., Forkel, S. J., Simmons, A., Vergani, F., Murphy, D. G., et al. (2011). A lateralized brain network for visuospatial attention. Nat Neurosci, 14, 1245-1246.

Thiebaut de Schotten, M., Ffytche, D. H., Bizzi, A., Dell'Acqua, F., Allin, M., Walshe, M., et al. (2011). Atlasing location, asymmetry and inter-subject variability of white matter tracts in the human brain with MR diffusion tractography. Neuroimage, 54, 49-59.

Thiebaut de Schotten, M., Kinkingnehun, S., Delmaire, C., Lehericy, S., Duffau, H., Thivard, L., et al. (2008). Visualization of disconnection syndromes in humans. Cortex, 44, 1097-1103.

Thiebaut de Schotten, M., Tomaiuolo, F., Aiello, M., Merola, S., Silvetti, M., Lecce, F., et al. (2014). Damage to white matter pathways in subacute and chronic spatial neglect: a group study and 2 single-case studies with complete virtual "in vivo" tractography dissection. Cereb Cortex, 24, 691-706.

Toepper, M., Markowitsch, H. J., Gebhardt, H., Beblo, T., Thomas, C., Gallhofer, B., et al. (2010). Hippocampal involvement in working memory encoding of changing locations: an fMRI study. Brain Res, 1354, 91-99.

Treisman, A. (1998). Feature binding, attention and object perception. Philos Trans R Soc Lond B Biol Sci, 353, 1295-1306.

Treisman, A. M., \& Gelade, G. (1980). A feature-integration theory of attention. Cogn Psychol, 12, 97-136.

Townsend, J. T., \& Ashby, F. G. (1982). Experimental test of contemporary mathematical models of visual letter recognition. J Exp Psychol Hum Percept Perform, 8, 834-854.

Tzourio-Mazoyer, N., Landeau, B., Papathanassiou, D., Crivello, F., Etard, O., Delcroix, N., et al. (2002). Automated anatomical labeling of activations in SPM using a macroscopic anatomical parcellation of the MNI MRI single-subject brain. Neuroimage, 15, 273-289.

Verdon, V., Schwartz, S., Lovblad, K. O., Hauert, C. A., \& Vuilleumier, P. (2010). Neuroanatomy of hemispatial neglect and its functional components: a study using voxel-based lesion-symptom mapping. Brain, 133, 880-894.

Watson, D. G., \& Humphreys, G. W. (1997). Visual marking: prioritizing selection for new objects by topdown attentional inhibition of old objects. Psychol Rev, 104, 90-122.

Watson, D. G., \& Humphreys, G. W. (2000). Visual marking: evidence for inhibition using a probe-dot detection paradigm. Percept Psychophys, 62, 471-481.

Wolfe, J. M. (1994). Visual search in continuous, naturalistic stimuli. Vision Res, 34, 1187-1195.

Woolsey, T. A., Hanaway, J., \& Gado, M. H. (2008). The brain atlas : a visual guide to the human central nervous system (3rd ed.). Hoboken, N.J.: Wiley.

Worsley, K. J. (2003). Developments in random field theory. In R.S.J.Frackowiak, K. J. Friston, C. Frith, R. Dolan, C. J. Price, S. Zeki, J. Ashburner, \& W. D. Penny (Eds.), Human Brain Function (2nd ed., pp. 881-886). London: Academic Press. 
Table 1. Patient's details: clinical and demographic data.

\begin{tabular}{|c|c|c|c|c|}
\hline & $\begin{array}{l}\text { Mean (or } \\
\text { number) }\end{array}$ & Std Deviation & $\begin{array}{c}\text { Minimum } \\
\text { value** }\end{array}$ & $\begin{array}{c}\text { Maximum } \\
\text { value*** }\end{array}$ \\
\hline Age (years) & 63.1 & 10.7 & 34 & 81 \\
\hline Sex (male/female) & $34 / 5$ & $\mathrm{~N} / \mathrm{A}$ & $\mathrm{N} / \mathrm{A}$ & $\mathrm{N} / \mathrm{A}$ \\
\hline Handedness (R/L) & $36 / 3$ & $\mathrm{~N} / \mathrm{A}$ & $\mathrm{N} / \mathrm{A}$ & $\mathrm{N} / \mathrm{A}$ \\
\hline Aetiology (S/CM/ND)* & $31 / 3 / 5^{*}$ & $\mathrm{~N} / \mathrm{A}$ & $\mathrm{N} / \mathrm{A}$ & $\mathrm{N} / \mathrm{A}$ \\
\hline Time post lesion (years) & 5.7 & 4.7 & 1 & 18 \\
\hline Lesion volume $\left(\mathrm{cm}^{3}\right)$ & 30.5 & 26.9 & 0.22 & 90.7 \\
\hline Lesion side $(\mathrm{R} / \mathrm{L} / \mathrm{B})$ & $16 / 8 / 15$ & $\mathrm{~N} / \mathrm{A}$ & $\mathrm{N} / \mathrm{A}$ & $\mathrm{N} / \mathrm{A}$ \\
\hline Corsi Block & 3.7 & 1.1 & $1(0)$ & $6(?)$ \\
\hline Digit span (forward) & 4.8 & 1.6 & $2(0)$ & $7(?)$ \\
\hline Digit span (backwards) & 2.6 & 1.2 & $0(0)$ & $5(?)$ \\
\hline ACT accuracy & 43.5 & 11.8 & $10(0)$ & $50(50)$ \\
\hline ACT/AFA left deficits & 2.4 & 5.0 & $0(0)$ & $22(25)$ \\
\hline ACT/AFA right deficits & 0.9 & 3.3 & $0(0)$ & $8(25)$ \\
\hline ACT/AIncA left deficits & 1.5 & 2.5 & $0(0)$ & $9(25)$ \\
\hline ATC/AIncA right deficits & 0.3 & 1.4 & $0(0)$ & $18(25)$ \\
\hline
\end{tabular}

* All patients included in this study were at chronic stage post diagnosis; out of 39 patients, three suffered from carbon monoxide poisoning and five from degenerative changes (three of these patients also suffered from different forms of unspecified vascular disease causing acquired focal brain lesions). Omitting carbon-monoxide patients from the analyses made little qualitative difference to the results. These patients were subsequently included in all analyses reported here to maximise statistical power. ${ }^{* *}$ minimum and maximum scores as measured in the studied group of stroke patients, numbers in brackets indicated minimum and maximum score for a given test; ACT, Apple Cancellation task; the maximum achievable 
score in the Apple Cancellation task is 50 (ACT accuracy). The cut-off for total numbers of target (full apples) omissions i.e. accuracy score is 40/50. Egocentric neglect is determined by whether patients miss targets (complete apples) on the left or right side of the page (asymmetry score calculated based on left- vs. right-side errors, ACT/AFA asymmetry score for full apples indicating either left or right deficits). Allocentric neglect is determined by whether patients make false positive responses by cancelling incomplete apples (distractors) where the gap is on either the right or left side of each apple, irrespective of the position of the (incomplete) apple on the page (asymmetry score calculated based on left- vs. right-side errors, AIncA asymmetry score for incomplete apples); B, bilateral; CM, carbon monoxide poisoning; ND, neurodegenerative changes; L, left; R, right; S, stroke; 
Table 2. Percentage errors by the patients and control participants.

\begin{tabular}{lcccccc}
\hline Search condition & \multicolumn{2}{l}{ Preview search } & \multicolumn{5}{c}{ Conjunction search } \\
\hline Display size & 4 & 8 & 16 & 4 & 8 & 16 \\
Patients contralesional & 1.5 & 5.7 & 12.3 & 2.7 & 10.3 & 15.2 \\
Patients ipsilesional & 0.9 & 3.1 & 8.1 & 1.5 & 6.2 & 9.3 \\
Controls & 0.5 & 3.9 & 5.2 & 0.8 & 5.6 & 8.7 \\
\end{tabular}


Table 3. Grey matter substrates of visual search.

\begin{tabular}{|c|c|c|c|c|c|}
\hline & Clt & r level & Voxel level & Coordinates & Brain Structure \\
\hline Mo & $\mathbf{P}_{\text {FWE }}$ & Size & Z-score & $\begin{array}{lll}X & Y & Z\end{array}$ & (location) \\
\hline
\end{tabular}

Model 1: overall conjunction search

$\begin{array}{lllllll}0.05 & 231 & 3.95 & \mathbf{3 4} & \mathbf{- 8 0} & \mathbf{3 6} & \text { Right } \mathrm{AG}\end{array}$

Model 2: overall preview search

$\begin{array}{lllllll}0.03 & 279 & 4.07 & \mathbf{2 8} & \mathbf{- 9 8} & \mathbf{1 0} & \text { Right MOG } \\ 0.002 & 466 & 4.00 & \mathbf{3 4} & \mathbf{- 7 6} & \mathbf{3 4} & \text { Right AG } \\ 0.02 & 283 & 3.78 & \mathbf{- 3 2} & \mathbf{- 9 4} & \mathbf{1 6} & \text { Left MOG }\end{array}$

Model 3: field effect in conjunction search (ipsi<contralesional)

$\begin{array}{lllllll}0.000 & 2480 & 4.37 & \mathbf{5 8} & \mathbf{- 2 4} & \mathbf{4 0} & \text { Right SMG extending into } \\ & & & & & \\ & & & & & & \text { postcentral gyrus } \\ 0.000 & 2495 & 3.71 & \mathbf{4 2} & \mathbf{1 0} & \mathbf{- 2 2} & \text { Right STG, MTG extending } \\ & & & & & & \\ & & & & & & \end{array}$

Abbreviations: AG, angular gyrus; MOG, middle occipital gyrus; MTG, middle temporal gyrus; SMG, supramarginal gyrus; STG, superior temporal gyrus. 
Table 4. White matter substrates of visual search.

\begin{tabular}{|c|c|c|c|c|c|}
\hline & Clt & r level & Voxel level & Coordinates & Brain Structure \\
\hline lod & $\mathbf{P}_{\mathbf{F W E}}$ & Size & Z-score & $\begin{array}{lll}X & Y & Z\end{array}$ & (location) \\
\hline
\end{tabular}

Model 1: overall conjunction search

$\begin{array}{lllllll}0.02 & 279 & 3.65 & \mathbf{3 0} & \mathbf{- 6 6} & \mathbf{2 0} & \text { Right IFOF, INTC }\end{array}$

Model 2: overall preview search

\begin{tabular}{|c|c|c|c|c|c|}
\hline 0.000 & 4141 & 4.70 & $18-48$ & 38 & Right IFOF, INTC, SLF \\
\hline & & 4.68 & $-26-66$ & 10 & Left IFOF, INTC, SLF \\
\hline
\end{tabular}

Model 3: field effect in conjunction search (ipsi<contralesional)

$\begin{array}{lllllll}0.000 & 2059 & 4.20 & \mathbf{4 4} & \mathbf{- 6} & \mathbf{3 4} & \text { Right SLF, INTC, }\end{array}$

Abbreviations: IFOF, inferior fronto-occipital fasciculus; INTC, internal capsule; SLF, superior longitudinal fasciculus. 
Conjunction Search

$\mathrm{H} \quad \mathrm{H}$

(H) $\mathrm{A} \quad \mathrm{H}$

A $\mathrm{H} \quad \mathrm{H}$

A $\quad$ A $\mathrm{H}$

A

A $\mathrm{H}$ H A
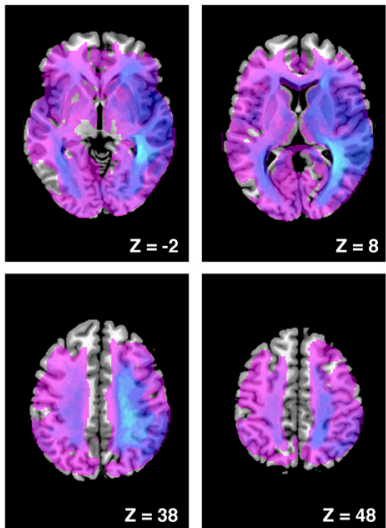

\section{Preview Search}

H H

H H

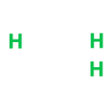

H

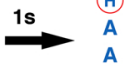

(H)

$\begin{array}{lll}\text { A } & \mathrm{H} & \mathrm{H}\end{array}$

A

H H

A H H A
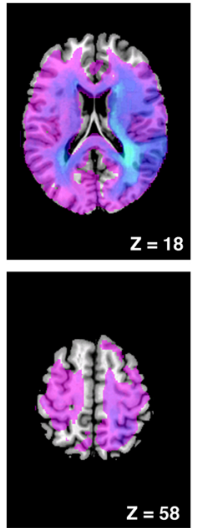

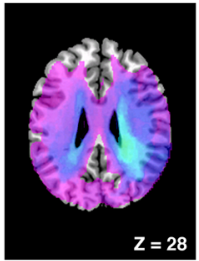

39

들

1 


\section{Control participants}
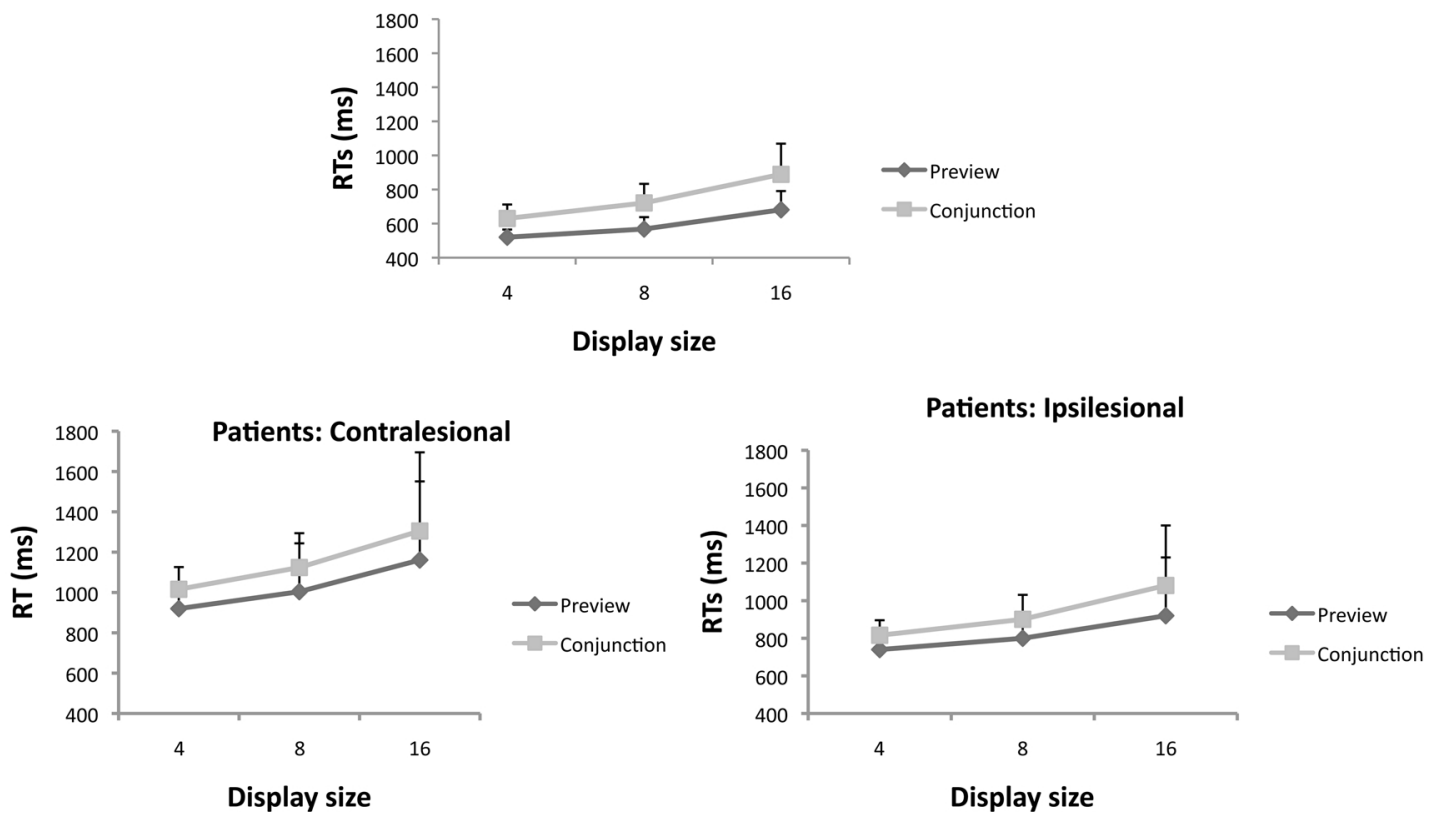

Display size 
A
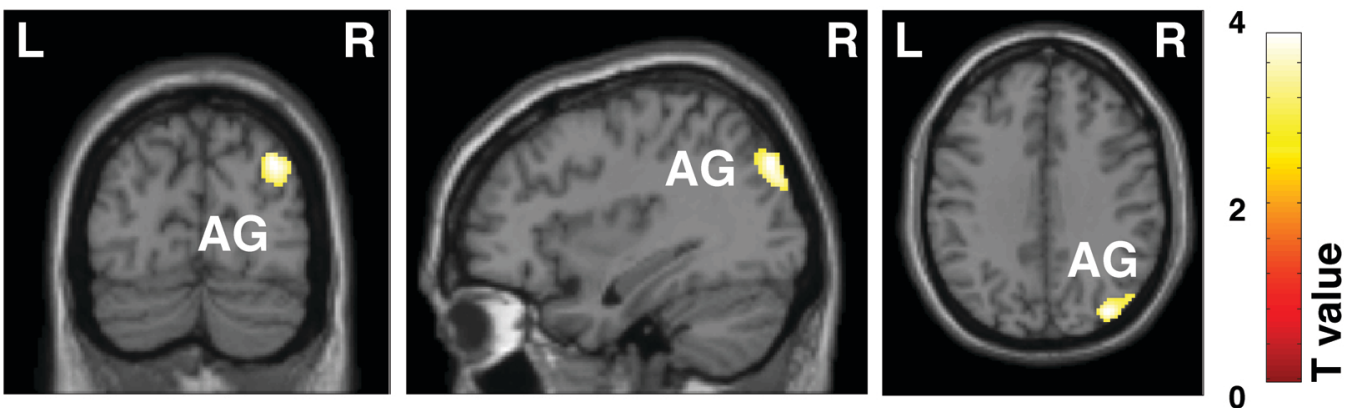

B
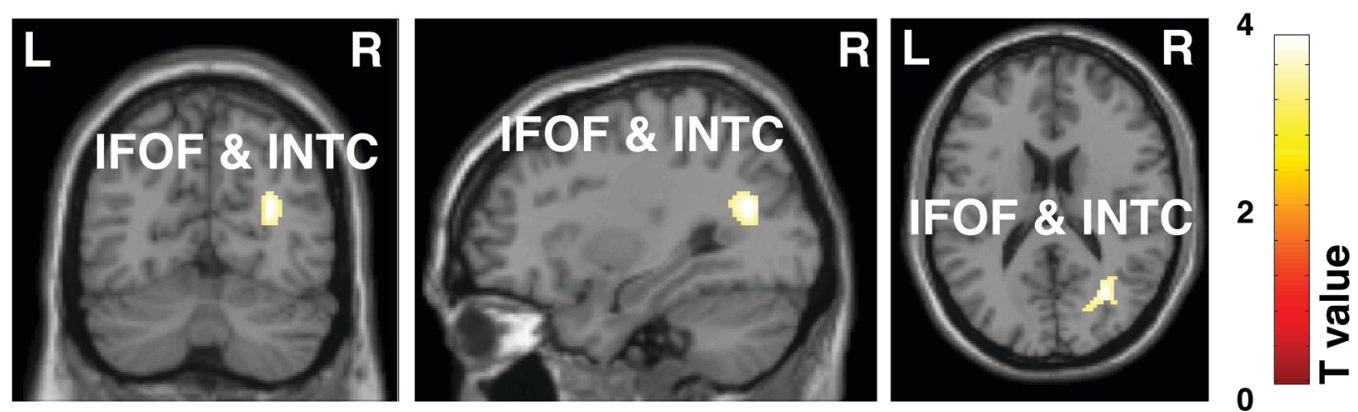

C

right IFOF \& INTC

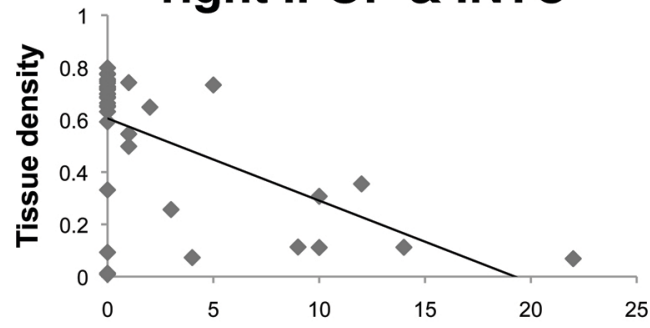

Egocentric neglect score

right IFOF \& INTC

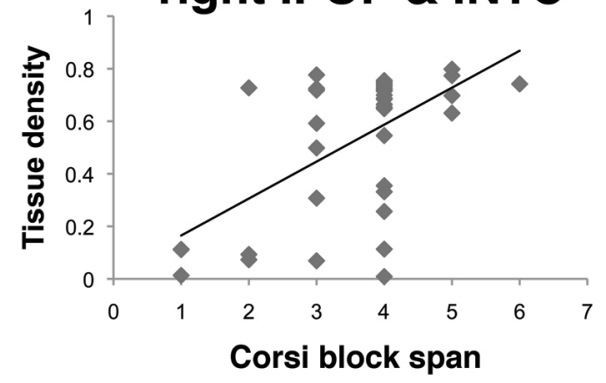



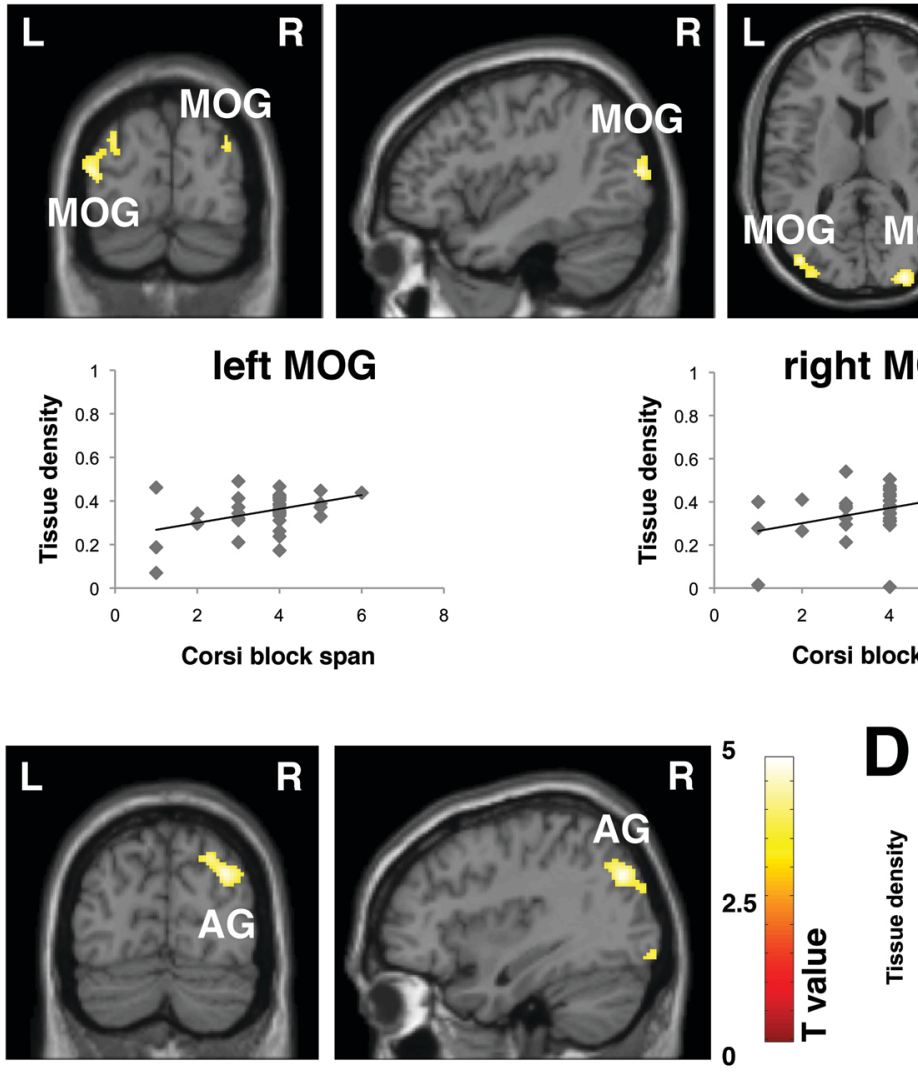

right $A G$

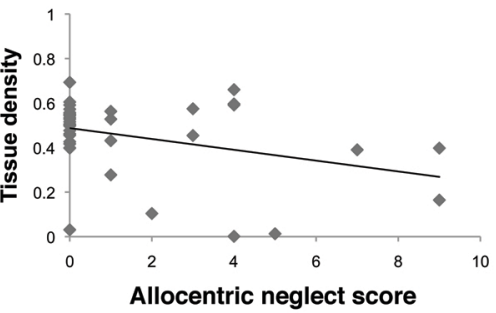

E
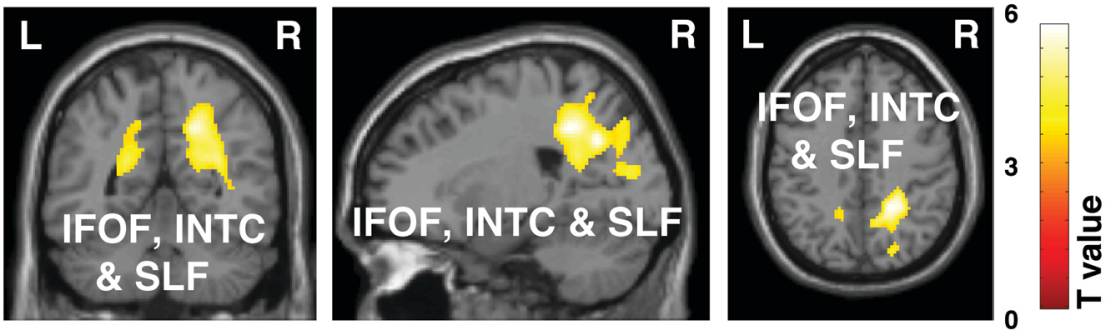
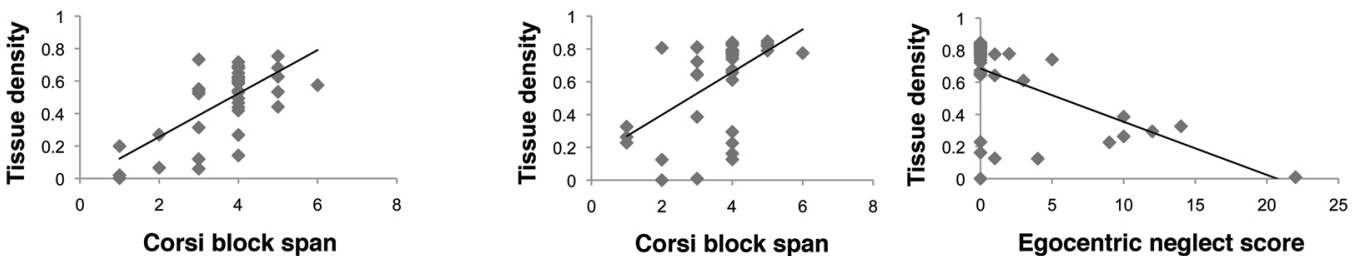

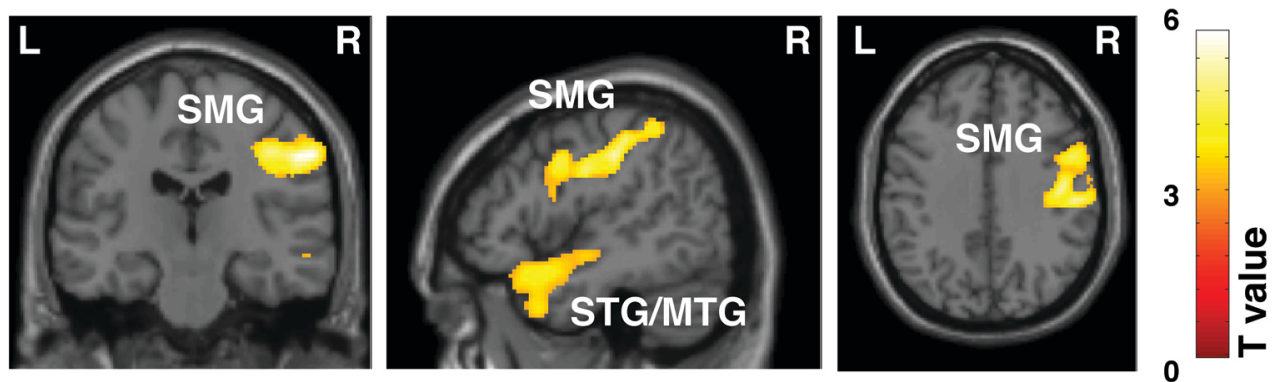

B

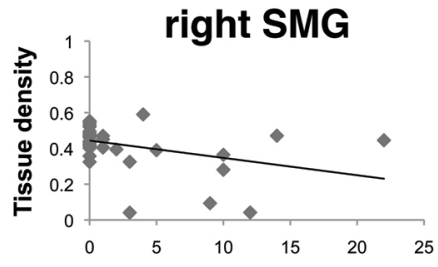

Egocentric neglect score

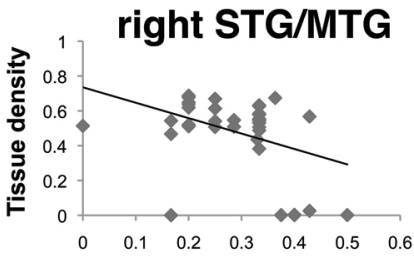

Forward-backward digit span/ summed spans right SMG

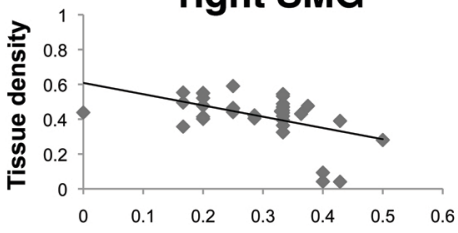

Forward-backward digit span/ summed spans

c
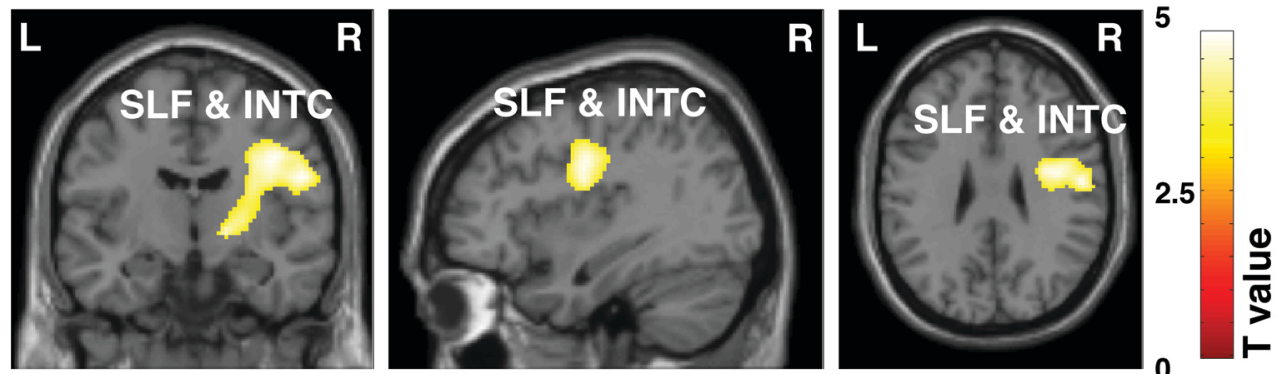